\title{
Managing Our Patients with Acute Primary Angle Closure: Are there Better Alternatives?
}

\author{
Clement CY Tham ${ }^{1-3}$ \\ ${ }^{1}$ Department of Ophthalmology \& Visual Sciences, The Chinese University of Hong Kong; \\ ${ }^{2}$ Hong Kong Eye Hospital, Kowloon; and ${ }^{3}$ Prince of Wales Hospital, Shatin, Hong Kong, China
}

Acute primary angle closure (APAC) remains an important disease in many parts of Asia, resulting in considerable morbidity and even irreversible blindness.

The three main challenges in the management of APAC are to:

- reduce intraocular pressure (IOP), and thus the symptoms, swiftly and safely

- prevent recurrence of APAC

- prevent progression to primary angle closure glaucoma (PACG).

Traditionally, IOP reduction has largely been achieved by both topical and systemic IOP-lowering drugs (stage 1 treatment), while the preventions of recurrence of APAC and progression to PACG have been partially achieved by laser peripheral iridotomy (stage 2 treatment). Recent evidence suggested that with this traditional approach, it may take more than 2 hours to achieve IOP control and symptomatic relief. ${ }^{1}$ Furthermore, up to $58 \%$ of Asian patients may progress to the chronic form of the disease. ${ }^{2}$ In view of these inadequacies, there has been a continuous search for more effective alternative treatments for APAC.

Anterior chamber paracentesis has been proposed as one possible alternative to drugs as stage 1 treatment for APAC. Lam et al described the successful application of immediate paracentesis as stage 1 treatment in a pilot series of 10 patients with APAC. ${ }^{3}$ In this issue of Asian Journal of Ophthalmology, Artini et al describe their use of paracentesis in a larger series of 45 patients with APAC. ${ }^{4}$ In addition to these published studies, Dr Paul Palmberg in Miami, USA, has also described his successful use of this technique in patients with APAC at several major international conferences (Palmberg P; 2011; personal communications).

Different techniques of paracentesis have been described. Lam et $\mathrm{al}^{3}$ and Palmberg performed their paracenteses at the slit lamp in the clinic, while Artini et al ${ }^{4}$ performed paracentesis under an operating microscope with the patient supine. Lam et $\mathrm{a}^{3}$ advocated

Correspondence: Prof Clement CY Tham, Department of Ophthalmology \& Visual Sciences, The Chinese University of Hong Kong, Hong Kong Eye Hospital, 147K Argyle Street, Kowloon, Hong Kong, China.

Tel: (852) 2762 3196;

E-mail: clemtham@cuhk.edu.hk the use of the $15^{\circ}$ disposable paracentesis knife, while Artini et al ${ }^{4}$ and Palmberg employed a 30-G needle. Lam et al ${ }^{3}$ administered systemic IOP-lowering drugs in addition to paracentesis, while Artini et al ${ }^{4}$ performed paracentesis without systemic drugs. Artini et al performed the laser peripheral iridotomy on the day after the paracentesis. ${ }^{4}$ In the author's experience, the advantage of immediate paracentesis is that the IOP can be quickly reduced, and corneal clarity returns very promptly, so laser iridotomy can often be performed on the same day.

While the simplicity and speed of immediate paracentesis remain attractive, we should remember that this procedure is still an invasive intervention with potentially serious complications. These complications may include endophthalmitis, suprachoroidal haemorrhage, decompression retinopathy, and sharp injury to any ocular structures that lie in the path of the knife or needle. Furthermore, there are also less invasive alternatives, such as argon laser peripheral iridoplasty (ALPI). ${ }^{1}$ To date, there has not been a randomized trial of sufficient scale and follow-up to properly compare immediate paracentesis to the alternative stage 1 treatments such as ALPI.

In addition to ALPI and paracentesis, corneal indentation may be a third alternative to drugs as a stage 1 treatment for APAC, ${ }^{5}$ while the role of laser iridotomy as a stage 2 treatment has recently been challenged by lens extraction. ${ }^{6}$ The search for the ideal treatment strategy for APAC is active and ongoing. Thanks to the intensive, continuing research efforts in APAC worldwide, and in the Asia-Pacific region in particular, we are edging ever closer to the Holy Grail.

\section{References}

1. Lam DS, Lai JS, Tham CC, Chua JK, Poon AS. Argon laser peripheral iridoplasty versus conventional systemic medical therapy in treatment of acute primary angle-closure glaucoma: a prospective, randomized, controlled trial. Ophthalmology. 2002; 109:1591-6.

2. Aung T, Ang LP, Chan SP, Chew PT. Acute primary angle-closure: long-term intraocular pressure outcome in Asian eyes. Am J Ophthalmol. 2001;131:7-12.

3. Lam DS, Chua JK, Tham CC, Lai JS. Efficacy and safety of immediate anterior chamber paracentesis in the treatment of acute 


\section{Editorial}

primary angle-closure glaucoma: a pilot study. Ophthalmology. 2002;109:64-70.

4. Artini W, Gondowiardjo TD, Afandi ES. Role of sequential treatment of paracentesis followed by laser peripheral iridotomy in acute primary angle closure. Asian J Ophthalmol. 2011;13;3-8.

5. Masselos K, Bank A, Francis IC, Stapleton F. Corneal indentation in the early management of acute angle closure. Ophthalmology. 2009;116:25-9.

6. Lam DS, Leung DY, Tham CC, et al. Randomized trial of early phacoemulsification versus peripheral iridotomy to prevent intraocular pressure rise after acute primary angle closure. Ophthalmology. 2008;115:1134-40. 